\title{
овеш Оасcess
}

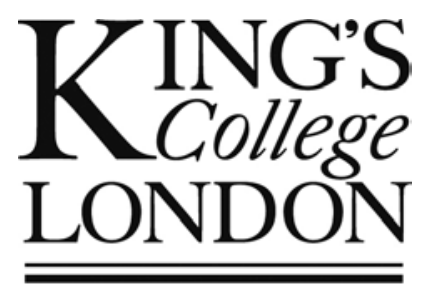

\section{King's Research Portal}

DOI:

10.1080/14737175.2019.1585243

Document Version

Peer reviewed version

Link to publication record in King's Research Portal

Citation for published version (APA):

Hoffmann, J., \& May, A. (2019). Neuromodulation for the treatment of primary headache syndromes. Expert Review of Neurotherapeutics, 19(3), 261-268. https://doi.org/10.1080/14737175.2019.1585243

\section{Citing this paper}

Please note that where the full-text provided on King's Research Portal is the Author Accepted Manuscript or Post-Print version this may differ from the final Published version. If citing, it is advised that you check and use the publisher's definitive version for pagination, volume/issue, and date of publication details. And where the final published version is provided on the Research Portal, if citing you are again advised to check the publisher's website for any subsequent corrections.

\section{General rights}

Copyright and moral rights for the publications made accessible in the Research Portal are retained by the authors and/or other copyright owners and it is a condition of accessing publications that users recognize and abide by the legal requirements associated with these rights.

- Users may download and print one copy of any publication from the Research Portal for the purpose of private study or research. - You may not further distribute the material or use it for any profit-making activity or commercial gain

- You may freely distribute the URL identifying the publication in the Research Portal

\section{Take down policy}

If you believe that this document breaches copyright please contact librarypure@kcl.ac.uk providing details, and we will remove access to the work immediately and investigate your claim. 


\title{
Neuromodulation for the treatment of primary headache syndromes
}

\author{
Jan Hoffmann ${ }^{1,2} \&$ Arne May $^{1}$ \\ ${ }^{1)}$ Department of Systems Neuroscience, University Medical Center Hamburg-Eppendorf, \\ Hamburg, Germany \\ ${ }^{2)}$ Basic and Clinical Neurosciences, Institute of Psychiatry, Psychology and Neuroscience, King's College, \\ London, United Kingdom
}

Keywords: Cluster headache, External trigeminal nerve stimulation, Headache, Migraine, Neuromodulation, Non-invasive vagal nerve stimulation, Occipital nerve stimulation, Sphenopalatine ganglion stimulation, Transcranial magnetic stimulation

Word count abstract: 200 words

Word count main text: 5006 words

References: 60

\section{Corresponding author}

Prof. Dr. med. Arne May

Department of Systems Neuroscience

University Medical Center Hamburg-Eppendorf

Martinistrasse 52

20246 Hamburg

Germany

Tel: +49-40-7410-59189

Fax: +49-40-7410-59955

a.may@uke.de 


\section{Abstract}

$\underline{\text { Introduction }}$

Neuromodulation techniques play an increasing role in the treatment of primary headaches. While initially reserved for refractory cases they are now increasingly taken into consideration in earlier treatment phases and in non-refractory situations. One of the main reasons of this paradigm shift is that most neuromodulation techniques are better tolerated as compared to the majority of pharmacological approaches. However, these techniques have their limitations that should be considered.

\section{Areas covered}

The review provides an overview of the available techniques and their therapeutic rationale as well as on the evidence for their efficacy and their limitations. The review covers these aspects for non-invasive vagal nerve stimulation, sphenopalatine ganglion stimulation, external trigeminal nerve stimulation, occipital nerve stimulation as well as single-pulse and repetitive-pulse transcranial magnetic stimulation.

\section{Expert commentary}

Most of the evidence is based on open-label studies. Sham devices used in controlled studies remain problematic as they either do not produce the paresthesias perceived during stimulation or induce some degree of stimulation. Invasive techniques require a surgical intervention with all the potential complications that may arise.

In summary some of the techniques provide an effective expansion of available treatment options but their indication should be thoroughly evaluated before treatment is considered. 


\section{Introduction}

Neuromodulation techniques are becoming increasingly important for the treatment of several primary headaches. With the availability of a range of invasive and non-invasive approaches and the increasing evidence on their efficacy, they represent a valuable expansion of the therapeutic armamentarium. From a patients' perspective these treatments are well accepted as patients increasingly seek nonpharmacological treatments given that currently available acute and preventive medications frequently induce unwanted side-effects that may lead to treatment discontinuation. Nevertheless, neuromodulation techniques are still seen as rather unconventional and their use is limited by the fact that in many countries most of the devices are not reimbursed by health care insurance. As their cost is usually relatively high, the long-term use of most of the devices exceeds the financial capabilities of most patients.

However, the lack of reimbursement is not simply based on the health care providers' responsibility to limit health care expenses. Clinical trials on these devices commonly have methodological shortcomings that are inherent to their mode of action. Initially many studies were uncontrolled due to the lack of adequate sham devices. While this problem has been solved by most of the manufacturers the utility of sham devices is limited as the majority of neuromodulation devices cause sensory disturbances (paresthesia or hypesthesia) during the stimulation, whereas the sham devices commonly do not induce these sensations. Some companies have addressed this problem by stimulating with modified stimulation parameters or subthreshold stimulations with lower amplitudes that do not induce sensory alterations but this approach bears the risk that these sham devices may already have a limited effect that could affect the difference to the verum group, thereby falsely rendering the treatment ineffective. Despite these difficulties, the quality of the data has improved substantially and slowly but steadily it becomes clearer which technique may offer a useful alternative and with technique does not. 
In this review we will provide an overview and an interpretation of the existing data on each of the neuromodulatory techniques currently available for the treatment of primary headaches.

\section{Non-invasive vagal nerve stimulation}

Non-invasive vagal nerve stimulation (nVNS) (GammaCore ${ }^{\circledR}$ ) has been developed for the acute and preventive treatment of migraine and cluster headache. In this stimulation procedure a small handheld device is placed on the neck on the ipsilateral side to the head pain; it is turned on by the patient who then increases the amplitude of stimulating current until a light tingling sensation is perceived on the skin. The device stimulates the area and automatically turns off after 120 seconds. Depending on the treatment protocol stimulation can be repeated after a brief pause on the same side, on the contralateral side for abortive treatment. In case of a preventive intervention, treatment is following a fixed schedule two to three times daily. The pathophysiological rationale is, that the externally applied current stimulates the vagal nerve. This stimulation is thought to reduce neuronal activity in the trigeminocervical complex of the brainstem(1), a key relay center that passes nociceptive information from the peripheral part of the trigeminovascular system to higher processing centers(2-4), as well as well as in the autonomic parasympathetic pathway that plays a pivotal role in cluster headache and other trigemino-autonomic cephalalgias(5). However, given the widespread distribution of the applied current and the very limited amount of solid experimental data, there is still substantial uncertainty of the influenced nociceptive pathways. The molecular mechanisms that are potentially affected remain unknown.

\section{$\underline{\text { nVNS in migraine }}$}

Since its development a large number of clinical trials have been conducted to prove its efficacy in migraine and cluster headache but unfortunately much of the available data is contradictory. For the 
acute treatment of migraine the device was first studied in an open-label design in which patients stimulated up to three consecutive attacks each with two right-sided stimulations of 120 seconds separated by an stimulation-free interval of 3 minutes within 20 minutes if the onset of mild or moderate pain(6). The results suggested efficacy with $64.6 \%$ of patients experiencing pain relief and $39.6 \%$ being pain free two hours after stimulation(6). Since the results were based on an uncontrolled study-design (no sham group), a randomized, sham-controlled trial (RCT) followed (PRESTO trial) to confirm this finding. However, the RCT showed efficacy at the 30 and 60 minute time points but failed to do so at the 120 minute time point which was the primary endpoint (pain free at two hours)(7).

The clinical trials to prove its efficacy in the preventive treatment of migraine shared the same fate. In the initial open-label trial (no sham group) a bilateral self-treatment of 20 minutes twice daily over 3 months led to a significant reduction of migraine and headache days in patients with episodic and chronic migraine(8) but these results could not be confirmed in the RCT that followed (EVENT study)(9). The sham-controlled EVENT study which involved a two-month randomized period followed by a sixmonth open-label phase clearly failed to reduce monthly migraine days in the randomized phase. Only at the last time point of the open-label phase a weak significance was observed although it is likely that this finding is artificial as only 15 patients reached this time-point and it may be assumed that dropped out were those that had no effect.

\section{$\underline{\text { nVNS in cluster headache }}$}

For the assessment of efficacy in the acute treatment of cluster headache the sham-controlled ACT1 trial was conducted(10). In this study patients stimulated unilaterally (side of pain) three consecutive times each lasting 120 seconds separated by a stimulation-free interval of one minute. Sham stimulation was performed applying a low-frequency $(0.1 \mathrm{~Hz})$ biphasic signal that does not stimulate the vagus nerve. The response (proportion of patients who achieved a pain intensity of zero or one on a five-point scale 
without the use of rescue medication) was assessed 15 minutes after stimulation. While the study failed to prove efficacy in the complete study population it revealed that nVNS was effective in the acute treatment of patients with episodic but not in those with chronic cluster headache. To confirm these findings a second RCT (ACT2 study) was conducted, which showed the same result using a slightly different primary endpoint (pain free at 15 minutes)(11). Again nVNS was only effective in the acute treatment of episodic but not in chronic cluster headache. While these findings were intensively discussed in the field as they are difficult to explain with the current understanding of the pathophysiology of cluster headache, the studies on the preventive treatment of cluster headache using the monoclonal antibodies against calcitonin gene-related peptide (CGRP) galcanezumab and fremanezumab follow the same pattern (i.e. efficacy in episodic but not in chronic cluster headache). However, it has to be considered that these were preventive treatment approaches whereas the nVNS findings were obtained in an acute treatment design.

For the efficacy of nVNS in the preventive treatment of chronic cluster headache only data derived from an open-label study (PREVA study) exists(12). In this study a right-sided stimulation of three consecutive stimulations of 120 seconds separated by stimulation-free intervals of five minutes, applied twice daily over four weeks as an add on-therapy to standard of care therapy, proved to be superior to standard of care therapy without additional nVNS. An RCT on the preventive treatment of episodic or chronic cluster headache does not yet exist.

$\underline{\text { nVNS in other trigeminoautonomic cephalalgias }}$

Tso et al.(13) explored the potential efficacy of nVNS in indomethacin-sensitive trigeminoautonomic cephalalgias (TAC), namely chronic paroxysmal hemicrania and hemicrania continua. All observed patients were responsive to indomethacin but either had to reduce or even discontinue the treatment due to adverse effects so that in this study in some patients nVNS was used as a monotherapy, in some 
as an add-on therapy to indomethacin used at the maximum tolerated dose. Of the nine patients with hemicrania continua and the six patients with chronic hemicrania continua had a significant improvement of their condition while only four patients ( 2 with hemicrania continua and two with chronic paroxysmal hemicrania) had no response to nVNS(13). Sham-controlled studies are clearly needed to confirm these findings.

\section{Sphenopalatine ganglion stimulation}

The sphenopalatine ganglion (SPG) stimulation (Pulsante ${ }^{\circledR}$ ) was developed initially for the acute treatment of cluster headache. In this neurostimulation technique a small electrode is implanted through the oral cavity into the close vicinity of the SPG. After the surgical intervention and a recovery period of four weeks, patients receive a handheld device to perform an on-demand activation of the electrode(14). To do so patients have to hold the device during an attack onto their cheek to activate the electrode and induce pain relief. The main advantage of this induction-based approach is that the battery is located in the handheld device and not in the electrode so that no surgical revisions are required for the exchange of batteries. The downside of this method is that patients have to hold the device onto their cheek during the entire stimulation period (15-20 minutes) which is not always easy given the excruciating severity of the headache.

The pathophysiological rationale of this technique is that the applied high-frequency stimulation of the SPG inhibits the neuronal activity of the parasympathetic arm of the trigeminoautonomic reflex which is thought to play an essential role in the pathophysiology of cluster headache $(4,5,15,16)$.

The clinical efficacy of SPG stimulation in cluster headache has been demonstrated in the shamcontrolled pathway $\mathrm{CH}-1$ study(17). In this study 28 patients with refractory chronic cluster headache used the handheld device during 30 attacks or until a maximum treating period of eight weeks was completed. Patients were instructed to stimulate 15 minutes during attacks that had at least moderate 
intensity and refrain from using acute medications in this period. The device randomly (1:1:1) delivered either a full stimulation, a subthreshold stimulation ( $85 \%$ of the amplitude that provoked a sensory perception) or no stimulation. The study reached its primary endpoint defined as pain-relief within 15 minutes of the start of neurostimulation. The probability of pain relief after 15 minutes reached $67.1 \%$ compared to $7.4 \%$ under sham stimulation while the probability of being pain free after 15 minutes of stimulation was $34.1 \%$ compared to $1.5 \%$ after sham stimulation. In 12 out of the 28 patients a reduction in attack frequency was observed. In these patients attack frequency was reduced by $88 \%$. In total the $\mathrm{CH}-1$ trial showed that $68 \%$ of the patients receiving full stimulation had either an acute response, a frequency response, or both. With respect to the observed adverse events, the majority of patients reported a loss of sensation in maxillary nerve regions that was mostly transient as $65 \%$ of the recorded events resolved within three months(17). A second RCT (NCT02168764) has been conducted in the United States to confirm these findings but the results have not been published yet.

The long-term follow-up of patients under SPG-stimulation revealed that the acute and preventive effects are consistent in the vast majority of patients over the entire observational period of 24 months(18). The same applies to the type of effect the SPG-stimulation had on a given patient. For example if the patient had only an acute but no preventive effect from SPG-stimulation he was likely to have only that acute effect after 24 months. The same applied for patients showing only a preventive effect or those showing both, an acute and a preventive effect(18). Beyond that the study showed that a substantial amount of patients that entered the study with a therapy-refractory chronic cluster headache switched to an episodic form allowing them to reduce or even discontinue their preventive medication(19). Taken together, based on the clinical data available at this point, the effect of SPGstimulation is effective and consistent making it an attractive therapeutic option in patients with therapy-refractory chronic cluster headache. 


\section{External trigeminal nerve stimulation}

The external trigeminal nerve stimulation (eTNS) has been developed for the acute and preventive treatment of migraine. In this technique a small neurostimulation device (Cefaly ${ }^{\circledR}$ device) is placed on the forehead and stimulations is performed with rectangular biphasic symmetrical pulses. For the acute treatment of migraine stimulation is performed with a pulse frequency of $100 \mathrm{~Hz}$ for 60 minutes, for the preventive treatment a frequency of $60 \mathrm{~Hz}$ is used and stimulation is performed daily for 20 minutes. The maximum stimulation intensity for both treatment types is $16 \mathrm{~mA}$. The precise mode of action is unknown but data from imaging studies based on FDG-PET and fMRI suggest functional changes in the orbitofrontal cortex and the rostral anterior cingulate cortex $(20,21)$.

Efficacy data for eTNS in the acute treatment of migraine relies on an open-label data study as an RCT does not exist. In the study published by Chou et al.(22), 30 patients suffering from an acute attack of migraine with or without aura, treated their attack for one hour with the Cefaly ${ }^{\circledR}$ device. Primary outcome parameter was the change in pain intensity after 1 hour compared to baseline. The intervention led to reduction of headache intensity by $57.1 \%$ compared to baseline (reduction of $3.22 \pm$ 2.40 on a VAS ranging from $0-10$ ). The effect remained significant one hour after the end of the stimulation period with none of the patients requiring rescue medication at this time point. Even after 24 hours only $65.4 \%$ of patients made use of a rescue medication. While the study clearly shows that eTNS may be effective in the acute treatment of migraine the data rely on an open-label study with all the inherent shortcomings of a study without a sham group. Therefore an RCT is needed to confirm these results.

In contrast for the preventive treatment of migraine a RCT does exist, the PREMICE study(23). In this study 67 patients were randomized with 34 patients receiving verum stimulation and 33 patients receiving sham stimulation. Compared to verum stimulation sham stimulation was performed using a lower pulse width of $30 \mu$ s (compared to $250 \mu$ s in verum), a frequency of $1 \mathrm{~Hz}$ (instead of $60 \mathrm{~Hz}$ ) and an 
intensity of $1 \mathrm{~mA}$ (instead of $16 \mathrm{~mA}$ ). In both groups daily stimulation sessions lasted 20 minutes. Efficacy assessment was performed 90 days after the initiation of treatment. Primary outcome measurements were change in monthly migraine days as well as the percentage of subjects having at least a $50 \%$ reduction in monthly migraine days at month three compared to the run-in baseline month. The study showed that monthly migraine days were reduced by $29.7 \%$ whereas in the sham group an increase of $4.9 \%$ was observed. While the difference between month three and the run-in month was significant, the difference between both groups failed to reach significance $(p=0.054)$ due to the high standard deviation of the data. In line with these findings the $50 \%$ responder rate was higher in the verum than in the sham group. In both study arms no adverse events were observed.

In summary the available data suggest that eTNS provides an effective treatment option for the preventive and perhaps also acute treatment of migraine. Nevertheless larger studies are clearly needed to confirm these results.

\section{Occipital nerve stimulation}

The occipital nerve stimulation (ONS) was initially developed for the treatment of cluster headache but its indication was later expanded to migraine. The rationale for stimulation of the occipital nerve is based on the fact that the trigeminal and occipital nerves converge in the trigeminocervical complex, in particular at the level of $\mathrm{C2}$, where they share the same nuclei(24). This anatomical situation allows a functional interaction and a mutual modulation of incoming signals of the trigeminal and occipital nerves before the nociceptive signals proceed to higher processing centers of the CNS(25-29). This modulating interaction is thought to be the basis for the efficacy of the (pharmacological) greater occipital nerve block(30,31). However, the exact effect of the neurostimulation of the occipital nerve and its influence on pain perception remains to be elucidated. 


\section{$\underline{\text { ONS in cluster headache }}$}

Several small open-label studies have suggested efficacy of ONS in chronic cluster headache(32-36) but to date no RCTs exist to confirm the obtained findings. In all of these studies patients experienced a significant reduction of attack frequency reaching in some patients up to $90 \%$ allowing many of them a reduction of their preventive medication. However, all of these studies had no sham control group so that results have to be interpreted with caution. Another shortcoming has long been the lack of longterm data in regard to efficacy and safety of the intervention. Recently data has been published that suggest that the observed effects are sustainable with observational periods of up to 9 years(33, 37-39). These studies focused on severely affected patients with refractory chronic cluster headache, but again, these studies are observational without a sham control group. A RCT that would address the long-term effect and safety does not yet exist.

\section{$\underline{\text { ONS in migraine }}$}

In contrast, RCT's investigating the efficacy of ONS in migraine do exist. In the large sham-controlled trial published by Silberstein et al. patients with chronic migraine were randomized in a 2:1 scheme with 105 patients allocated to the active and 52 patients to the sham group(40). Electrodes were implanted unior bilaterally depending on the distribution of headache. Stimulation was performed using the IPG Genesis ${ }^{\circledast}$ system (St. Jude Medical Neuromodulation). Patients allocated to the sham group received a sham programmer that did not communicate with the IPG Genesis ${ }^{\circledR}$ system. Following a 1-month baseline period treatment was performed over 12 weeks. Primary outcome parameter was the mean daily intensity with a responder being a patient that had a reduction of at least $50 \%$. The study did not meet its primary endpoint. From all patients allocated to the active group only $17.1 \%$ (18 patients) were classified as responders while in the sham group this was the case in $13.5 \%$ ( 7 patients) of patients. In contrast, the authors observed a significant reduction in headache days and migraine-related disability. 
In the open-label extension phase of the same study all patients that decided to continue received active stimulation over an additional period of 40 weeks(41). In total 157 patients were included in the final intention to treat analysis. In this extension study $47.8 \%$ of patients achieved a $50 \%$ response and headache frequency was significantly reduced(41). However, despite the positive outcome of this extension study the results have to be interpreted with caution due to the open-label design.

In addition to these considerations regarding the efficacy of ONS in cluster headache and migraine ONS is not unproblematic as complications such as infections and lead migration are relatively common(42) leading to a temporal withdrawal of its approval by regulatory authorities in some countries.

\section{Single-pulse transcranial magnetic stimulation}

Single-pulse transcranial magnetic stimulation (sTMS) consists of a single magnetic pulse applied to the scalp and underlying cortex via a portable device.

The thought that sTMS could be effective in the acute treatment of migraine with aura originated with the observation made in in vivo experiments that a wave of cortical neuronal depolarization, the cortical spreading depression (CSD), which is likely to be the underlying cause of the aura(43), could potentially depolarize meningeal nociceptors and thereby induce migraine pain(44). Based on this assumption Holland et al. showed that STMS is able to disrupt $\operatorname{CSD}(45,46)$. These observations let to the rationale that sTMS could be able to abort a migraine attack if sTMS was applied once the aura initiated.

This hypothesis was tested in a randomized, double-blind, parallel-group, sham-controlled trial(47). In this study 201 patients were allocated to either active stimulation (sTMS) or sham. Upon stimulation, the device (Cerena Transcranial Magnetic Stimulator, Neuralieve) delivered a single magnetic field pulse of $0.9 \mathrm{~T}$ with a rise time of $180 \mu$ s and a total pulse length of under $1 \mathrm{~ms}$ and patients were instructed to apply 2 pulses delivered $30 \mathrm{~s}$ apart below the occipital bone. Patients initiated stimulation as soon as 
possible after aura began and within $1 \mathrm{~h}$ of aura onset. Primary endpoint was the proportion of patients that were pain free at $2 \mathrm{~h}$ post-treatment. The study revealed that the proportion of patients being painfree at $2 \mathrm{~h}$ was significantly higher in those receiving active stimulation (39\%) compared to those in the sham group (22\%).

In addition to its utility in the acute treatment of migraine attacks, sTMS has been studied as a preventive treatment (ESPOUSE study)(48). The rationale for expanding the study population to patients with and without aura was based on the observation that TMS can alter modulatory thalamocortical pathways suggesting a potential efficacy regardless of the presence of migraine aura(46). In this prospective study patients stimulated with the SpringTMS device (eNeura) over the occiput delivering four pulses twice daily over 3 months with the same parameters used in the preceding study described above. In addition to this preventive protocol, patients were allowed to treat an acute attack with 3 consecutive pulses which could be repeated up to 2 times if necessary. Primary endpoint was the mean reduction in headache days from baseline to the 4-week period extending between weeks 9-12. As a comparator the authors chose a performance goal (statistically derived placebo response) which was based on the placebo responses observed in one device study and two clinical trials evaluating the efficacy of topiramate in the preventive treatment of migraine.

The study revealed a significant reduction of headache days from baseline compared to the performance goal. Likewise, the $50 \%$ responder rate of $46 \%$ in the responder group was significantly higher than the $20 \%$ of the performance goal. As in the previous study only few adverse events and no severe adverse events were observed(48).

Taken together, current evidence suggests that sTMS may be an effective and well-tolerated treatment option for the acute and preventive treatment of migraine. 


\section{Repetitive-pulse transcranial magnetic stimulation}

The rationale behind using repetitive-pulse transcranial magnetic stimulation (rTMS) for the preventive treatment of migraine was basically the same as for sTMS, namely the modulation of cortical excitability(49-51). While this seems a solid approach as most preventive medications inhibit cortical excitability(52), the influence of rTMS on cortical excitability appears to be more complex as the modulating influence depends on the pulse frequency. While rTMS at high frequency $(20 \mathrm{~Hz})$ increases cortical excitability, stimulation at low frequency $(1 \mathrm{~Hz})$ has the opposite effect $(53,54)$.

Information obtained from several clinical studies that aimed at assessing its efficacy for the preventive treatment of migraine have provided inconsistent results that are even contradicting the findings of the studies described above. In addition to the importance of using the appropriate stimulation frequency, the location of the stimulation seems to be at least equally important.

In the sham-controlled study performed by Misra et al.(55), 100 patients were randomized to either active rTMS or sham. Active stimulation consisted of 600 pulses delivered at $10 \mathrm{~Hz}$ in 10 trains over $412 \mathrm{~s}$ applied over the motor cortex at the hot spot of the right abductor digiti minimi. Primary endpoint was the reduction in headache frequency and severity of at least $50 \%$ after 1 month of daily stimulation. The study showed that compared to baseline headache frequency was reduced $78.7 \%$ in the active group compared to $33.3 \%$ in the sham group and headache severity was improved by $76.6 \%$ in the active group compared to $27.1 \%$ in the sham group. Therefore, the data suggest that $10 \mathrm{~Hz} r T M S$ at this particular site seems to be effective for the preventive treatment of migraine.

Brighina et al.(56) made similar observations in their sham-controlled study when rTMS was applied with a frequency of $20 \mathrm{~Hz}$ (10 trains of $2 \mathrm{~s}$ duration) at the dorsolateral prefrontal cortex. However, the findings of this study have to be taken with caution as they are based on the observations made on 11 patients. 
In contrast to these findings, Teepker et al.(57) analyzed the efficacy of low frequency rTMS for the preventive treatment of migraine in a sham-controlled study. Active stimulation was provided over 5 consecutive days in 2 trains consisting of 500 pulses delivered at a frequency of $1 \mathrm{~Hz}$ over the vertex. Primary endpoint was the reduction of migraine attacks during the 8-week follow-up compared to baseline. The study showed that while the number of migraine attacks was reduced significantly in the active group, the difference to the response observed in the sham group failed to reach statistical significance. These findings suggest that low-frequency stimulation is not effective in the preventive treatment of migraine. However, if the lack of efficacy is due to an inappropriate stimulation frequency or the wrong site of stimulation remains to be elucidated.

In line with these results, Conforto et al.(58) showed that rTMS applied in 23 sessions over 8 weeks on the dorsolateral prefrontal cortex had no significant effect on headache days in patients with chronic migraine. Interestingly, in this study stimulation was performed at high frequency $(10 \mathrm{~Hz})$ therefore contradicting the findings obtained when stimulation was performed over the motor cortex at the hot spot of the right abductor digiti minimi.

Summarizing the available data on rTMS, the data shows that high frequency rTMS $(10 \mathrm{~Hz})$ over the motor cortex at the hot spot of the right abductor digiti minimi is effective in preventing migraine attacks while low frequency stimulation $(1 \mathrm{~Hz})$ over the vertex is not. Data on rTMS over the dorsolateral prefrontal cortex is inconsistent as two studies using high frequency stimulation showed opposing results. Given this body of evidence further sham-controlled studies that would identify the appropriate site and frequency of rTMS are clearly needed before definitive conclusions on the efficacy in preventing migraine attacks can be drawn.

\section{Conclusion}


Neurostimulation techniques have remarkably expanded the therapeutic toolbox of the neurologist treating primary headaches, in particular migraine and cluster headache. Their main advantage compared to current pharmacological acute and preventive treatment options is their superior sideeffect profile. However, regarding their efficacy many studies have significant limitations as some of them have an open-label design or a problematic sham device. Beyond that, most studies do not assess the efficacy of the blinding by asking patients at the end of the study if they assume they were in the treatment or the sham arm of the study. Based on data obtained exclusively from randomized, shamcontrolled trials, efficacy in primary headaches has been proven for most described devices. In detail, for cluster headache, nVNS has been proven effective in the acute treatment of episodic cluster headache attacks while SPG stimulation is effective in the acute and preventive treatment. In migraine, sTMS has been proven effective in the acute treatment of migraine and high-frequency rTMS significantly reduced headache frequency and severity. The current body of evidence clearly suggests that more randomized, sham-controlled studies are needed to confirm some of the positive results observed in open-label trials.

\section{Expert commentary}

While neuromodulation techniques play an increasingly important role as an expansion to currently available pharmacological treatments, evidence of most of the techniques remain problematic, the main reason being the difficulty in designing an adequate sham device that would mimic noises and perceptions during stimulation without inducing a clinically meaningful effect. Unfortunately, with the creation and improvement of adequate sham devices the data proving efficacy of some neuromodulation techniques has become less convincing so that the initially high expectations could not be met. Nevertheless most of these techniques have now earned their place in the treatment of primary 
headaches, in particular in refractory cases. Nevertheless, research activities in this area will have to focus on an improvement of sham-devices where this is possible. Beyond that further randomized, sham-controlled studies are required to confirm positive results seen in open-label trials. In addition, it may be assumed that investigations regarding their efficacy have not yet reached the end of the road as new therapeutic areas, as for example their utility in indomethacin-sensitive headaches, are likely to be revealed in the near future.

\section{Five-year view}

It may be assumed that research and development of neurostimulation devices will continue on a high pace over the next years. The most significant challenge of these studies will be the development of improved sham-devices as this has been the main shortcoming of current studies and probably a significant reason for the frequently conflicting results.

It can be expected that those devices exclusively tested in open-label studies will be studied for the efficacy in randomized, sham-controlled trials. Beyond that novel devices with entirely different mechanisms of action may become available $(59,60)$. Finally, the expansion of clinical trials on other primary headaches such as the indomethacin-responsive headaches is expected as preliminary results of some of the devices revealed promising results and these headache syndromes frequently lead to a significant reduction of quality of life as treatment options are limited and commonly associated with side-effects that require their discontinuation.

\section{Key issues}


- Neurostimulation techniques expand the therapeutic armamentarium of primary headaches, in particular migraine and cluster headache.

- Non-invasive vagal nerve stimulation (nVNS) has been proven effective in the acute treatment of cluster headache attacks in episodic cluster headache. Randomized-controlled trials for the acute and preventive treatment failed to prove an efficacy.

- Exploratory studies suggest that nVNS could be effective in the treatment of indomethacinresponsive headaches.

- Sphenopalatine ganglion (SPG) stimulation is effective in the acute and preventive treatment of chronic cluster headache.

- External trigeminal nerve stimulation (eTNS) has been tested in an RCT for the preventive treatment of migraine but failed to be effective when compared to placebo.

- Occipital nerve stimulation (ONS) has been shown to be ineffective in chronic migraine as the RCT failed to meet its primary endpoint (reduction in attack severit $\$ 5 \%$ ). While the authors observed a significant reduction in headache days a new RCT is required until efficacy can be assumed. For its efficacy in cluster headache many open-label trials but no RCTs exist. A convincing conclusion regarding its clinical efficacy in cluster headache is therefore not possible.

- Single-pulse transcranial magnetic stimulation has been proven effective for the acute treatment of migraine. For its efficacy in the preventive treatment of migraine no shamcontrolled RCT exists.

- Repetitive transcranial magnetic stimulation has been proven effective in an RCT if stimulation is provided at a high frequency $(10 \mathrm{~Hz})$. In contrast low-frequency stimulation $(1 \mathrm{~Hz})$ has not been proven effective. 


\section{Conflict of Interest}

Jan Hoffmann is consulting for/served on advisory boards for Allergan, Autonomic Technologies Inc. (ATI), Chordate Medical AB, Eli Lilly, Hormosan Pharma, Novartis and Teva. He received honoraria for speaking from Allergan, Autonomic Technologies Inc. (ATI), Chordate Medical AB, Novartis and Teva. He serves as Associate Editor for Cephalalgia and the Journal of Orofacial Pain and Headache.

Arne May reports no conflict of interest. He serves as the Editor-in-chief for Cephalalgia. He received unrestricted scientific grants from Electrocore and Chordate Medical AB paid to the University Medical Center Hamburg-Eppendorf.

ORCID

Jan Hoffmann 0000-0002-2103-9081

Arne May 0000-0002-3499-1506

\section{References}

[1] Akerman S, Simon B, Romero-Reyes M. Vagus nerve stimulation suppresses acute noxious activation of trigeminocervical neurons in animal models of primary headache. Neurobiology of Disease. 2017;102:96-104.

*Preclinical study that demonstrates the potential mechanism of action of $\mathrm{nVNS}$ in primary headaches 
[2] Akerman S, Holland PR, Goadsby PJ. Diencephalic and brainstem mechanisms in migraine. Nat Rev Neurosci. 2011;12:570-84.

[3] Goadsby PJ, Holland PR, Martins-Oliveira M, Hoffmann J, Schankin C, Akerman S. Pathophysiology of Migraine: A Disorder of Sensory Processing. Physiological Reviews. 2017;97:553-622. **Extensive review of the current knowledge on the pathophysiology of migraine

[4] Hoffmann J, Baca SM, Akerman S. Neurovascular mechanisms of migraine and cluster headache. Journal of Cerebral Blood Flow \& Metabolism. 2017:271678X17733655.

[5] Hoffmann J, May A. Diagnosis, pathophysiology, and management of cluster headache. The Lancet Neurology. 2017.

[6] Barbanti P, Grazzi L, Egeo G, Padovan AM, Liebler E, Bussone G. Non-invasive vagus nerve stimulation for acute treatment of high-frequency and chronic migraine: an open-label study. The Journal of Headache and Pain. 2015;16:61.

[7] Tassorelli C, Grazzi L, de Tommaso M, Pierangeli G, Martelletti P, Rainero I, et al. Noninvasive vagus nerve stimulation as acute therapy for migraine: The randomized PRESTO study. Neurology. 2018;91:e364-e73. 
*RCT that demonstrates that nVNS does not provide a sustained headache relief in the acute treatment of migraine

[8] Kinfe TM, Pintea B, Muhammad S, Zaremba S, Roeske S, Simon BJ, Vatter H. Cervical noninvasive vagus nerve stimulation (nVNS) for preventive and acute treatment of episodic and chronic migraine and migraine-associated sleep disturbance: preliminary findings from a prospective observational cohort study. The Journal of Headache and Pain. 2015;16:101.

[9] Silberstein SD, Calhoun AH, Lipton RB, Grosberg BM, Cady RK, Dorlas S, et al. Chronic migraine headache prevention with noninvasive vagus nerve stimulation: The EVENT study. Neurology. 2016;87:529-38.

* RCT demonstrating that nVNS is ineffective in reducing monthly migraine days in chronic migraine

[10] Silberstein SD, Mechtler LL, Kudrow DB, Calhoun AH, McClure C, Saper JR, et al. Non-Invasive Vagus Nerve Stimulation for the ACute Treatment of Cluster Headache: Findings From the Randomized, Double-Blind, Sham-Controlled ACT1 Study. Headache. 2016;56:1317-32.

**First RCT to demonstrate that nVNS is effective in the acute treatment of episodic but not of chronic cluster headache 
[11] Goadsby PJ, de Coo IF, Silver N, Tyagi A, Ahmed F, Gaul C, et al. Non-invasive vagus nerve stimulation for the acute treatment of episodic and chronic cluster headache: A randomized, doubleblind, sham-controlled ACT2 study. Cephalalgia. 2018;38:959-69.

[12] Gaul C, Diener H-C, Silver N, Magis D, Reuter U, Andersson A, et al. Non-invasive vagus nerve stimulation for PREVention and Acute treatment of chronic cluster headache (PREVA): A randomised controlled study. Cephalalgia. 2015;36:534-46.

[13] Tso AR, Marin J, Goadsby PJ. Noninvasive vagus nerve stimulation for treatment of indomethacin-sensitive headaches. JAMA Neurology. 2017;74:1266-7.

[14] Schoenen J, Jensen RH, Lantéri-Minet M, Láinez MJA, Gaul C, Goodman AM, et al. Stimulation of the sphenopalatine ganglion (SPG) for cluster headache treatment. Pathway $\mathrm{CH}-1$ : A randomized, shamcontrolled study. Cephalalgia. 2013;33:816-30.

**RCT demonstrating the efficacy of SPG-stimulation in the acute and preventive treatment of cluster headache

[15] Spencer SE, Sawyer WB, Wada H, Platt KB, Loewy AD. CNS projections to the pterygopalatine parasympathetic preganglionic neurons in the rat: a retrograde transneuronal viral cell body labeling study. Brain Research. 1990;534:149-69. 
[16] Fontaine D, Santucci S, Lanteri-Minet M. Managing cluster headache with sphenopalatine ganglion stimulation: a review. J Pain Res. 2018;11:375-81.

[17] Lainez M, Jensen R, May A, Lanteri-Minet M, Gaul C, Goodman A, et al. Pathway CH-1 Study: Sphenopalatine Ganglion (SPG) Stimulation for Acute Treatment of Chronic Cluster Headache (CCH) Initial Experience (S36.002). Neurology. 2012;78:S36.002.

[18] Jürgens TP, Barloese M, May A, Láinez JM, Schoenen J, Gaul C, et al. Long-term effectiveness of sphenopalatine ganglion stimulation for cluster headache. Cephalalgia. 2017;37:423-34.

[19] Barloese MCJ, Jürgens TP, May A, Lainez JM, Schoenen J, Gaul C, et al. Cluster headache attack remission with sphenopalatine ganglion stimulation: experiences in chronic cluster headache patients through 24 months. The Journal of Headache and Pain. 2016;17:67.

[20] Magis D, D'Ostilio K, Thibaut A, Pasqua VD, Gerard P, Hustinx R, et al. Cerebral metabolism before and after external trigeminal nerve stimulation in episodic migraine. Cephalalgia. 2017;37:88191. 
[21] Russo A, Tessitore A, Esposito F, Di Nardo F, Silvestro M, Trojsi F, et al. Functional Changes of the Perigenual Part of the Anterior Cingulate Cortex after External Trigeminal Neurostimulation in Migraine Patients. Frontiers in Neurology. 2017;8.

[22] Chou DE, Gross GJ, Casadei CH, Yugrakh MS. External Trigeminal Nerve Stimulation for the Acute Treatment of Migraine: Open-Label Trial on Safety and Efficacy. Neuromodulation: Technology at the Neural Interface. 2017:n/a-n/a.

[23] Schoenen J, Vandersmissen B, Jeangette S, Herroelen L, Vandenheede M, Gérard P, Magis D. Migraine prevention with a supraorbital transcutaneous stimulator: A randomized controlled trial. Neurology. 2013;80:697-704.

* RCT providing evidence for the efficacy of eTNS for the preventive treatment of migraine

[24] Angus-Leppan H, Lambert G, Michalicek J. Convergence of Occipital Nerve and Superior Sagittal Sinus Input in the Cervical Spinal Cord of the Cat. Cephalalgia. 1997;17:625-30.

[25] Bartsch T, Goadsby PJ. Stimulation of the greater occipital nerve induces increased central excitability of dural afferent input. Brain. 2002;125:1496-509. 
[26] Bartsch T, Goadsby PJ. Increased responses in trigeminocervical nociceptive neurons to cervical input after stimulation of the dura mater. Brain. 2003;126:1801-13.

[27] Busch V, Jakob W, Juergens T, Schulte-Mattler W, Kaube H, May A. Functional connectivity between trigeminal and occipital nerves revealed by occipital nerve blockade and nociceptive blink reflexes. Cephalalgia. 2006;26:50-5.

[28] Busch V, Jakob W, Juergens T, Schulte-Mattler W, Kaube H, May A. Occipital nerve blockade in chronic cluster headache patients and functional connectivity between trigeminal and occipital nerves. Cephalalgia. 2007;27:1206-14.

[29] Le Doaré K, Akerman S, Holland PR, Lasalandra MP, Bergerot A, Classey JD, et al. Occipital afferent activation of second order neurons in the trigeminocervical complex in rat. Neuroscience Letters. 2006;403:73-7.

[30] Ambrosini A, Vandenheede M, Rossi P, Aloj F, Sauli E, Pierelli F, Schoenen J. Suboccipital injection with a mixture of rapiachd long-acting steroids in cluster headache: A double-blind placebo-controlled study. Pain. 2005;118:92-6. 
[31] Leroux E, Valade D, Taifas I, Vicaut E, Chagnon M, Roos C, Ducros A. Suboccipital steroid injections for transitional treatment of patients with more than two cluster headache attacks per day: a randomised, double-blind, placebo-controlled trial. The Lancet Neurology. 2011;10:891-7.

[32] Magis D, Allena M, Bolla M, De Pasqua V, Remacle J-M, Schoenen J. Occipital nerve stimulation for drug-resistant chronic cluster headache: a prospective pilot study. The Lancet Neurology. 2007;6:314-21.

[33] Magis D, Gérard P, Schoenen J. Invasive occipital nerve stimulation for refractory chronic cluster headache: what evolution at long-term? Strengths and weaknesses of the method. The Journal of Headache and Pain. 2016;17:8.

[34] Burns B, Watkins L, Goadsby PJ. Treatment of medically intractable cluster headache by occipital nerve stimulation: long-term follow-up of eight patients. The Lancet. 2007;369:1099-106.

[35] Burns B, Watkins L, Goadsby PJ. Treatment of intractable chronic cluster headache by occipital nerve stimulation in 14 patients. Neurology. 2009;72:341-5.

[36] Fontaine D, Christophe Sol J, Raoul S, Fabre N, Geraud G, Magne C, et al. Treatment of refractory chronic cluster headache by chronic occipital nerve stimulation. Cephalalgia. 2011;31:1101-5. 
[37] Miller S, Watkins L, Matharu M. Treatment of intractable chronic cluster headache by occipital nerve stimulation: a cohort of 51 patients. European Journal of Neurology. 2017;24:381-90.

[38] Fontaine D, Blond S, Lucas C, Regis J, Donnet A, Derrey S, et al. Occipital nerve stimulation improves the quality of life in medically-intractable chronic cluster headache: Results of an observational prospective study. Cephalalgia. 2017;37:1173-9.

[39] Leone M, Cecchini AP, Messina G, Franzini A. Long-term occipital nerve stimulation for drugresistant chronic cluster headache. Cephalalgia. 2017;37:756-63.

[40] Silberstein SD, Dodick DW, Saper J, Huh B, Slavin KV, Sharan A, et al. Safety and efficacy of peripheral nerve stimulation of the occipital nerves for the management of chronic migraine: Results from a randomized, multicenter, double-blinded, controlled study. Cephalalgia. 2012;32:1165-79.

[41] Dodick DW, Silberstein SD, Reed KL, Deer TR, Slavin KV, Huh B, et al. Safety and efficacy of peripheral nerve stimulation of the occipital nerves for the management of chronic migraine: Long-term results from a randomized, multicenter, double-blinded, controlled study. Cephalalgia. 2015;35:344-58. 
[42] Martelletti P, Jensen $\mathrm{RH}$, Antal A, Arcioni R, Brighina F, de Tommaso M, et al. Neuromodulation of chronic headaches: position statement from the European Headache Federation. J Headache Pain. 2013;14:86.

[43] Hadjikhani N, Sanchez Del Rio M, Wu O, Schwartz D, Bakker D, Fischl B, et al. Mechanisms of migraine aura revealed by functional MRI in human visual cortex. Proc Natl Acad Sci U S A. 2001;98:4687-92.

[44] Bolay H, Reuter U, Dunn AK, Huang Z, Boas DA, Moskowitz MA. Intrinsic brain activity triggers trigeminal meningeal afferents in a migraine model. Nat Med. 2002;8:136-42.

[45] Holland PR, Schembri CT, Fredrick JP, Goadsby PJ. Transcranial magnetic stimulation for the treatment of migraine aura. Neurology. 2009;72:A250 (abstract IN4-1.005).

[46] Andreou AP, Holland PR, Akerman S, Summ O, Fredrick J, Goadsby PJ. Transcranial magnetic stimulation and potential cortical and trigeminothalamic mechanisms in migraine. Brain. 2016;139:2002-

14. 
[47] Lipton RB, Dodick DW, Silberstein SD, Saper JR, Aurora SK, Pearlman SH, et al. Single-pulse transcranial magnetic stimulation for acute treatment of migraine with aura: a randomised, doubleblind, parallel-group, sham-controlled trial. The Lancet Neurology. 2010;9:373-80.

**RCT demonstrating the efficacy of sTMS in acute treatment of migraine

[48] Starling AJ, Tepper SJ, Marmura MJ, Shamim EA, Robbins MS, Hindiyeh N, et al. A multicenter, prospective, single arm, open label, observational study of sTMS for migraine prevention (ESPOUSE Study). Cephalalgia. 2018;38:1038-48.

**RCT proving the efficacy of sTMS in the preventive treatment of migraine

[49] Wassermann EM, Lisanby SH. Therapeutic application of repetitive transcranial magnetic stimulation: a review. Clinical Neurophysiology. 2001;112:1367-77.

[50] Kobayashi M, Pascual-Leone A. Transcranial magnetic stimulation in neurology. The Lancet Neurology. 2003;2:145-56.

[51] Maeda F, Keenan JP, Tormos JM, Topka H, Pascual-Leone A. Modulation of corticospinal excitability by repetitive transcranial magnetic stimulation. Clinical Neurophysiology. 2000;111:800-5. 
[52] Ayata C, Jin H, Kudo C, Dalkara T, Moskowitz MA. Suppression of cortical spreading depression in migraine prophylaxis. Ann Neurol. 2006;59:652-61.

[53] Gangitano M, Valero-Cabré A, Tormos JM, Mottaghy FM, Romero JR, Pascual-Leone Á. Modulation of input-output curves by low and high frequency repetitive transcranial magnetic stimulation of the motor cortex. Clinical Neurophysiology. 2002;113:1249-57.

[54] Romero JR, Anschel D, Sparing R, Gangitano M, Pascual-Leone A. Subthreshold low frequency repetitive transcranial magnetic stimulation selectively decreases facilitation in the motor cortex. Clinical Neurophysiology. 2002;113:101-7.

[55] Misra UK, Kalita J, Bhoi SK. High-rate repetitive transcranial magnetic stimulation in migraine prophylaxis: a randomized, placebo-controlled study. Journal of Neurology. 2013;260:2793-801.

[56] Brighina F, Piazza A, Vitello G, Aloisio A, Palermo A, Daniele O, Fierro B. rTMS of the prefrontal cortex in the treatment of chronic migraine: a pilot study. Journal of the Neurological Sciences. 2004;227:67-71.

[57] Teepker M, Hötzel J, Timmesfeld N, Reis J, Mylius V, Haag A, et al. Low-frequency rTMS of the vertex in the prophylactic treatment of migraine. Cephalalgia. 2010;30:137-44. 
[58] Conforto AB, Amaro E, Gonçalves AL, Mercante JP, Guendler VZ, Ferreira JR, et al. Randomized, proof-of-principle clinical trial of active transcranial magnetic stimulation in chronic migraine. Cephalalgia. 2014;34:464-72.

[59] Yarnitsky D, Volokh L, Ironi A, Weller B, Shor M, Shifrin A, Granovsky Y. Nonpainful remote electrical stimulation alleviates episodic migraine pain. Neurology. 2017;88:1250-5.

[60] Wilkinson D, Ade KK, Rogers LL, Attix DK, Kuchibhatla M, Slade MD, et al. Preventing Episodic Migraine With Caloric Vestibular Stimulation: A Randomized Controlled Trial. Headache. 2017;57:106587. 